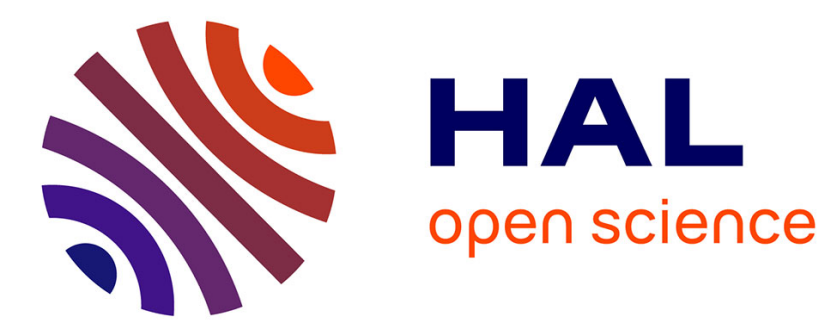

\title{
INTERACTIONS OF IMPURITIES WITH A MOVING GRAIN BOUNDARY
}

\author{
C. Bauer
}

\section{To cite this version:}

C. Bauer. INTERACTIONS OF IMPURITIES WITH A MOVING GRAIN BOUNDARY. Journal de Physique Colloques, 1975, 36 (C4), pp.C4-333-C4-338. 10.1051/jphyscol:1975433 . jpa-00216339

\section{HAL Id: jpa-00216339 https://hal.science/jpa-00216339}

Submitted on 1 Jan 1975

HAL is a multi-disciplinary open access archive for the deposit and dissemination of scientific research documents, whether they are published or not. The documents may come from teaching and research institutions in France or abroad, or from public or private research centers.
L'archive ouverte pluridisciplinaire HAL, est destinée au dépôt et à la diffusion de documents scientifiques de niveau recherche, publiés ou non, émanant des établissements d'enseignement et de recherche français ou étrangers, des laboratoires publics ou privés. 


\title{
INTERACTIONS OF IMPURITIES WITH A MOVING GRAIN BOUNDARY
}

\author{
C. I. BAUER \\ Department of Metallurgy \& Materials Science \\ Carnegie-Mellon University \\ Pittsburgh, Pennsylvania, USA
}

\begin{abstract}
Résumé. - La plupart des théories développées, pour interpréter l'interaction des impuretés avec un joint de grain en mouvement, supposent une ségrégation uniforme le long d'un joint de grains plan. Quand la vitesse du joint augmente, l'excès d'impuretés au joint exerce une force résistante sur le joint, jusqu'au moment où la force résistante ne peut plus contrebalancer la force motrice : le joint se libère alors de ses impuretés.

Dans ce travail, les hypothèses d'une répartition latérale uniforme des impuretés et de la planéité du joint de grains sont modifiées pour permettre à la fois une điffusion parallèle au joint et latérale des impuretés, dans le voisinage du joint de grains.

On a trouvé qu'entre les deux stades habituels (traînage des impuretés par le joint et libération du joint de ses impuretés), existe un stade important où sont instables à la fois la forme plane du joint et la répartition latérale uniforme des impuretés au joint. L'hypothèse suivante est avancée : dans ce régime instable, les joints de grains pourraient présenter tout un spectre de morphologies relativement complexes; la connaissance de ces morphologies conduirait à la première description complète du processus de séparation du joint de ses impuretés et à une meilleure compréhension de phénomènes plus complexes, comme le durcissement des solutions solides, la croissance des grains et la recristallisation secondaire.
\end{abstract}

\begin{abstract}
Most theories developed to explain interaction of impurities with a moving grain boundary involve a uniform excess impurity concentration distributed along a planar grain boundary. As boundary velocity increases, the excess impurities exert a net drag force on the boundary until a level is reached whereat the drag force no longer can balance the driving force and breakaway of the boundary from these impurities occurs. In this investigation, assumptions of a uniform lateral impurity profile and a planar grain boundary shape are relaxed by allowing both forward and lateral diffusion of impurities in the vicinity of a grain boundary. It is found that the two usual regions (drag of impurities by, and breakaway of a planar grain boundary) are separated by an extensive region wherein a uniform lateral impurity profile and a planar grain boundary shape are unstable. It is suspected that, in this unstable region, grain boundaries assume a spectrum of more complex morphologies and that elucidation of these morphologies can provide the first definitive description of the breakaway process and insight to more complex phenomena such as solid-solution strengthening, grain growth and secondary recrystallization.
\end{abstract}

1. Introduction. - Since grain boundary migration represents one of the most common types of solid state reaction, it has been the subject of many experimental and theoretical investigations. The object of most investigations is to interrelate macroscopic grain boundary velocity $v$ to a given set of material parameters, the most important of which are temperature $T$, impurity concentration $c$, and applied driving force $F$. Functionally, $v$ is related to $F$ by the expression

$$
v=M F
$$

where $M$ denotes macroscopic mobility. In turn, $M$ may be resolved into an intrinsic component $M_{\mathrm{I}}$ and an extrinsic component $M_{\mathrm{II}}$, which are related to $M$ by the expression

$$
1 / M=1 / M_{\mathrm{I}}+1 / M_{\mathrm{II}}
$$

The quantity $M_{I}$ is associated with transfer of matrix atoms from one grain to another by a diffusional process and is independent of impurity concentration, whereas $M_{\text {II }}$ is usually a complicated function of $T, c$ and $F$. It is recognized that the great variety of experimental results is generally due to the sensitive manner by which $M_{\mathrm{II}}$ depends on extrinsic variables. Consequently, it is important to understand processes associated with $M_{\text {II }}$ and thereby gain further insight to the complex phenomenon of grain boundary migration.

The most successful theories of grain boundary migration to date have been advanced by Licke and Detert [1], Cahn [2] and Lücke and Stiuwe $[3,4]$. These theories are based on the concept of interaction of lattice impurities with a moving grain boundary. This interaction is characterized by an interaction energy which varies with distance from a planar grain boundary. As a result, an excess (or deficit) concentration of impurities may be associated with the boundary, defined by an excess concentration profile. This profile is symmetric with respect to boundary position for a stationary boundary but becomes asymmetric when the boundary is in motion. This asymmetry produ- 
ces a drag force on the boundary so that concomitant (extrinsic) grain boundary velocity is controlled by diffusion of impurities normal to the plane of the boundary. As velocity increases, excess impurity concentration decreases while asymmetry increases and, as a result, the drag force first increases with velocity (due to greater increase of asymmetry) but then gradually decreases (due to greater decrease of net excess impurity concentration). At sufficiently large velocities, the impurities can not exert sufficient drag force to balance the driving force (the net force on the boundary must vanish in steady state) and breakaway of the boundary from the excess impurities occurs. Thereafter, (intrinsic) grain boundary velocity is not affected by the presence of impurities.

The events of the aforementioned discussion are represented schematically in figure 1 , wherein variation of excess impurity concentration is plotted as a function of (perpendicular) distance from a grain boundary for various boundary veloci-
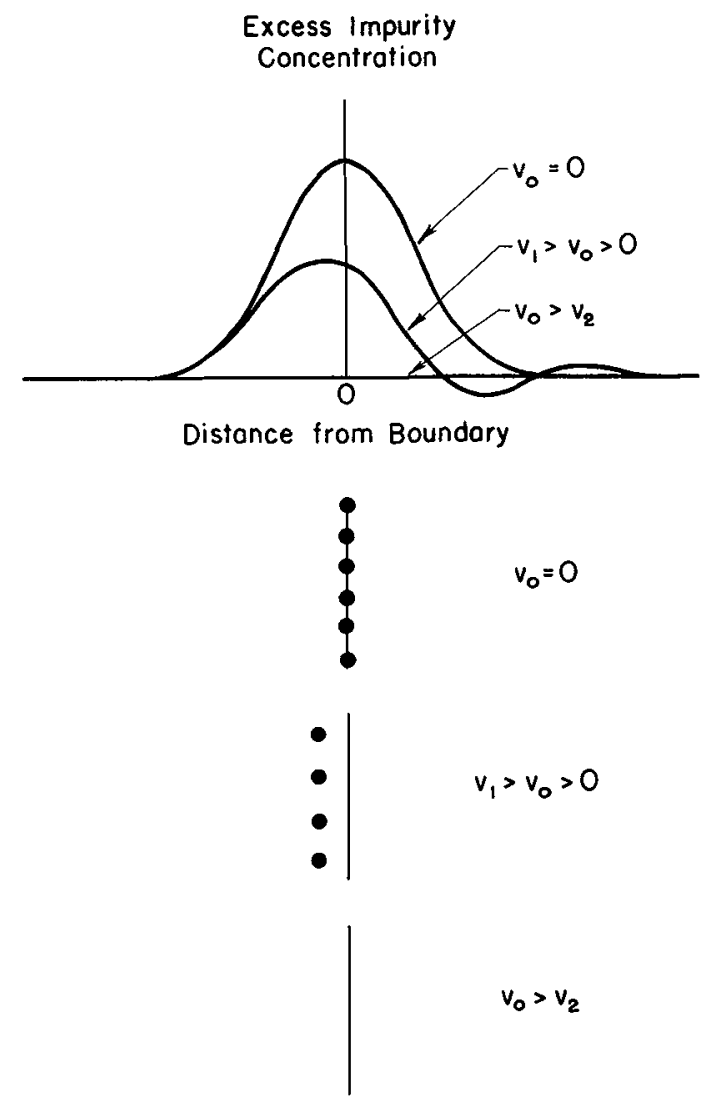

FIG. 1. - Variation of excess impurity concentration as a function of (perpendicular) distance from a grain boundary for various boundary velocities. The concentration profile is symmetric when macroscopic boundary velocity $v_{0}$ is either zero or greater than the breakaway velocity $v_{2}$, but is skewed when $v_{1}>v_{0}>0$, where $v_{1}$ denotes minimum velocity at which a planar grain boundary shape or a uniform lateral impurity concentration profile becomes unstable. In addition, an atomistic interpretation is illustrated schematically wherein the number (concentration) of impurity atoms is proportional to the net area under the excess concentration curve and the position of the impurity atoms corresponds to the center of gravity of this area. ties. The concentration profile is symmetric when macroscopic boundary velocity $v_{0}$ is either zero or greater than the breakaway velocity $v_{2}$, but is skewed when $v_{1}>v_{0}>0$, where $v_{1}$ denotes minimum velocity at which a planar grain boundary shape or a uniform lateral impurity concentration profile becomes unstable as considered in following sections. In addition, an atomistic interpretation is illustrated schematically wherein the number (concentration) of impurity atoms is proportional to the net area under the excess concentration curve and the position of the impurity atoms corresponds to the center of gravity of this area. Accordingly, the extent of lag of the impurities from the boundary provides a measure of the asymmetry of the impurity distribution and vice versa. In this context, the entire problem of grain boundary migration reduces to dermination of the correct impurity profile normal to the plane of a grain boundary.

The principal difficulty with the aforementioned model is that the boundary is forced to maintain a planar configuration and/or the excess impurity concentration is forced to be independent of position along the boundary. These conditions do obtain when grain boundary surface energy is infinite (forcing the boundary to be planar) or when diffusion coefficient of impurities parallel to the boundary is infinite (instantaneously smoothing out fluctuations of the excess impurity concentration). For realistic values of material parameters, however, retention of these constraints is not justified and entirely new concepts must be introduced in order to provide a correct interpretation of grain boundary migration.

The purpose of this paper is to review methods by which the aforementioned constraints are relaxed and thereafter emphasize necessary modifications of present-day concepts of grain boundary migration kinetics. The grain boundary model is described and relevant equations affecting boundary motion are defined in the following section. Thereafter, results stemming therefrom are presented and discussed in Sec. 3. Lastly, principal results and significance of this investigation are summarized in Sec. 4.

2. The Grain Boundary Model. - In order to incorporate features mentioned in the preceeding section into a grain boundary model, it is necessary to relax the assumption of a planar grain boundary shape and to allow both forward and lateral diffusion of impurities. Thus a two-dimensional grain boundary model is adopted as illustrated in figure 2 . In this model boundary displacement $\xi(x$, $t$ ) is expressed as a continuous function of position along the boundary $x$ and time $t$ by the expression

$$
\xi(x, t)=v_{0} t+\Delta \xi(x, t),
$$




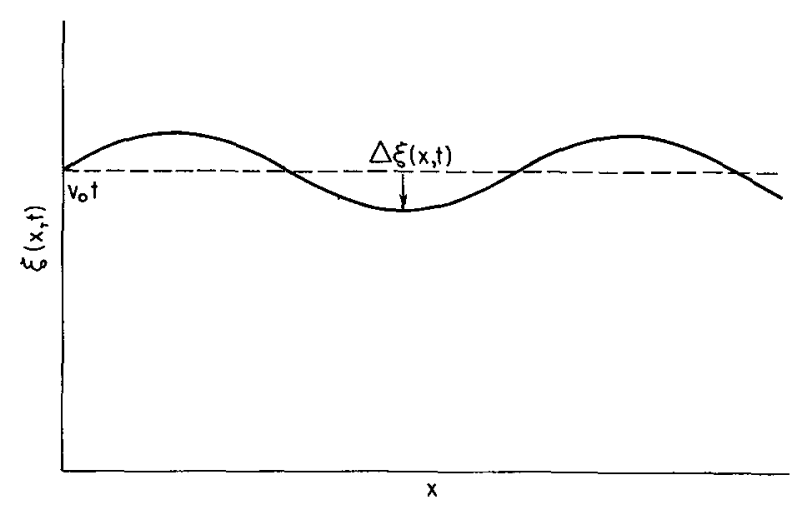

FIG. 2. - Grain boundary displacement $\xi(x, t)$ as a function of distance along spatial coordinate $x$. The quantity $\xi(x, t)$ is expressed at time $t$ by the sum of the displacement of a planar boundary moving at constant (macroscopic) velocity $v_{0}$ as $v_{0} t$ and a perturbation term representing deviation from a planar configuration $\Delta \xi(x, t)$; i. e., $\xi(x, t)=v_{0} t+\Delta \xi(x, t)$.

where $v_{\mathrm{o}}$ denotes average (macroscopic) boundary velocity and $\Delta \xi(x, t)$ denotes local displacement of the boundary from a planar configuration at position $x$ and time $t$. Likewise, excess concentration of impurities at the boundary ; i. e., only that concentration due to the presence of the boundary, is expressed by

$$
c(x, t)=c_{0}+\Delta c(x, t),
$$

where $c_{0}$ denotes average excess concentration (atomic fraction) of impurities at the boundary and $\Delta c(x, t)$ denotes deviation of the concentration from that for a planar boundary at position $x$ and time $t$. Although $c_{0}$ may be a complicated function of $v_{0}$, Eqs. (2.1) and (2.2) provide a general description of boundary shape and concentration profile under steady-state conditions.

In general, $\Delta \xi(x, t)$ and $\Delta c(x, t)$ are coupled to one another and must be.obtained through simultaneous solution of appropriate force and flux equations, except for the trivial case wherein both are equal to zero so that planar grain boundary shape and uniform lateral impurity concentration profile obtain, as assumed in Refs. [1-4]. Appropriate force and flux balances involving $\Delta \xi(x, t)$ and/or $\Delta c(x, t)$ have been considered by Roy and Bauer [5] and lead to a set of coupled differential equations which, under steady-state conditions, are given by

$$
D_{\perp} \partial^{2} \Delta \xi / \partial x^{2}-\left(v_{0} / \alpha\right) \Delta c=0
$$

and

$$
D_{\|} \partial^{2} \Delta c / \partial x^{2}+c_{0} v_{0} \partial^{2} \Delta \xi / \partial x^{2}=0,
$$

where $D_{\perp}$ and $D_{\|}$denote diffusion coefficients of impurities perpendicular and parallel to the grain boundary respectively and $\alpha$ denotes a dimensionless constant given by $\gamma / N_{\kappa} T$, where $N$ denotes number of atoms per unit boundary area, $\kappa$ denotes
Boltzmann's constant and $\gamma$ denotes macroscopic grain boundary surface energy. In effect, Eq. (2.3) represents a balance between the capillary force and local variation of the impurity drag force whereas Eq. (2.4) represents a balance between the diffusive and convective fluxes of impurities. Both equations are valid only under perturbation conditions wherein values of $\Delta \xi$ and $\Delta c$ are assumed to be sufficiently small.

Solutions to non-steady state equivalents of Eqs. (2.3) and (2.4) have been obtained by Roy and Bauer assuming periodic functions for $\Delta \xi$ and $\Delta c$ which are characterized by wave number $k$, where $k=2 \pi / \lambda$ and $\lambda$ denotes (common) wavelength of these functions. In so doing, it is found that fluctuations of either boundary shape or concentration profile may grow or decay, depending on values of a characteristic reciprocal relaxation time $q^{ \pm}$. Knowledge of various boundary shapes and concentration profiles may be gleaned by examination of velocity and wave number dependence of $q^{ \pm}$, as displayed in figure 3 . The negative root of

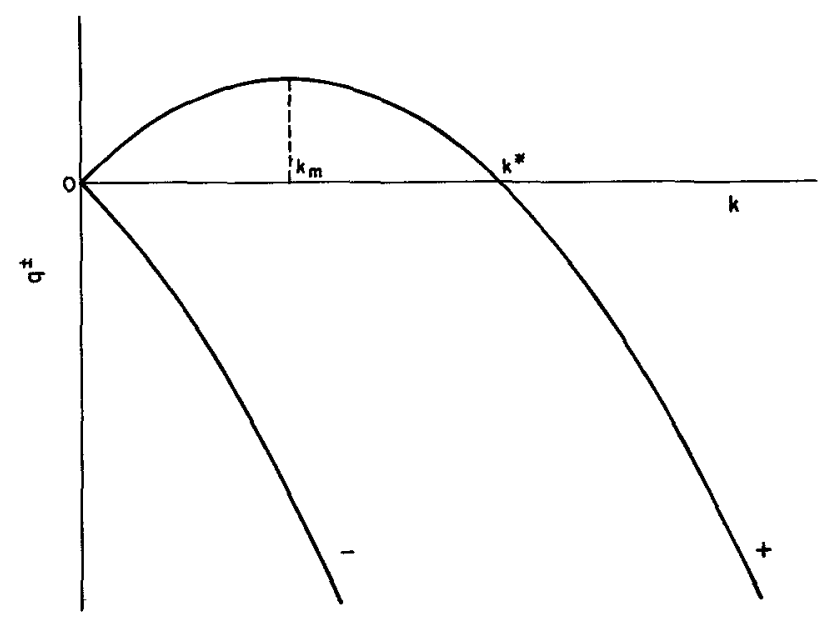

FIG. 3. - Variation of reciprocal relaxation time $q \pm$ as a function of wave number $k$. The quantity $q^{-}$is always negative indicating decay of all fluctuations whereas $q^{+}$is positive for $k<k^{*}$ and negative for $k>k^{*}$ indicating growth of fluctuations characterized by $k<k^{*}$ and decay of fluctuations characterized by $k>k^{*}$. The quantity $k_{\mathrm{m}}$ denotes the wave number of fluctuations which grow with maximum velocity.

$q^{\ddagger}$ is negative for all positive values of $k$ suggesting existence of a transient component, whereas the positive root can assume either positive or negative values, depending on specific values of $k$ and magnitude of $v_{0}$. Steady-state behavior is defined as the locus for $q^{ \pm}=0$, which is given by the expression

$$
k^{*}=v_{0}\left(\beta D_{\perp} D_{||}\right)^{-1 / 2},
$$

where $\beta=\alpha / c_{0}=\gamma / N c_{0} \kappa T$. Equation (2.5) specifies that, for a given macroscopic grain boundary velocity, a fluctuation of boundary shape and/or lateral concentration profile characterized by $k>k^{*}$ will decay whereas a fluctuation charac- 
terized by $k<k^{*}$ will grow, as depicted in figure 4 . Large diffusion coefficients of impurities near the boundary, a large surface tension, or a small concentration of impurities tend to stabilize the grain boundary. For a given boundary velocity, fluctuations characterized by wave number $k_{\mathrm{m}}$ corresponding to the maximum value of $q^{+}$ (cf. Fig. 3), will grow most rapidly.

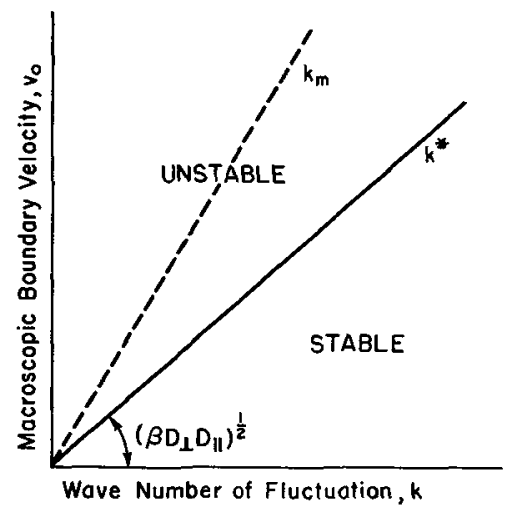

FIG. 4. - Macroscopic grain boundary velocity $v_{0}$ versus wave number of shape or concentration fluctuation $k$. Solid line separates regions wherein fluctuations can grow and decay; e. g., $k^{*}=\left(\beta D_{\perp} D_{\|}\right)^{-1 / 2} v_{0}$, whereas dashed line denotes wave number $k_{m}$ which characterizes maximum growth rate.

Steady-state amplitude ratio of the fluctuations is given by Roy and Bauer by the expression

$$
\Delta c / \Delta \xi=-c_{0} k^{*}\left(\beta D_{\perp} / D_{||}\right)^{1 / 2} .
$$

Although stability is governed by the product $\beta D_{\perp} D_{||}$(cf. Eq. 2.5) relative amplitudes of shape and conçentrations profiles are governed by the ratio $\beta D_{\perp} / D_{\|}$(cf. Eq. 2.6). Accordingly, a large value of $D_{\perp}$ (small extrinsic grain boundary drag) favors concentration redistribution without appreciable shape change whereas a large value of $D$ favors shape change without appreciable concentration redistribution. These two cases are illustrated schematically in figure 5 . The negative sign in Eq. (2.6) indicates that concentration build up occurs where the boundary lags the average displacement (where impurity drag is greatest and vice versa). Implications and ramifications of Eqs. (2.5) and (2.6) are considered in the following section.

3. Discussion. - The preceeding analysis demonstrates that, for any value of $v_{0}$, fluctuations of grain boundary shape and/or lateral impurity concentration profile for a sufficiently small wave number tend to grow, thus producing shape and/or concentration instability. In reality, however, the concept of instability is only meaningful when a given fluctuation grows to sufficient size over the time duration of an experiment; i. e., $q^{+}>k_{\mathrm{d}} v_{0}$, where $k_{\mathrm{d}}=2 \pi / \lambda_{\mathrm{d}}$ and $\lambda_{\mathrm{d}}$ denotes a physical dimension of the specimen (normally grain size). The
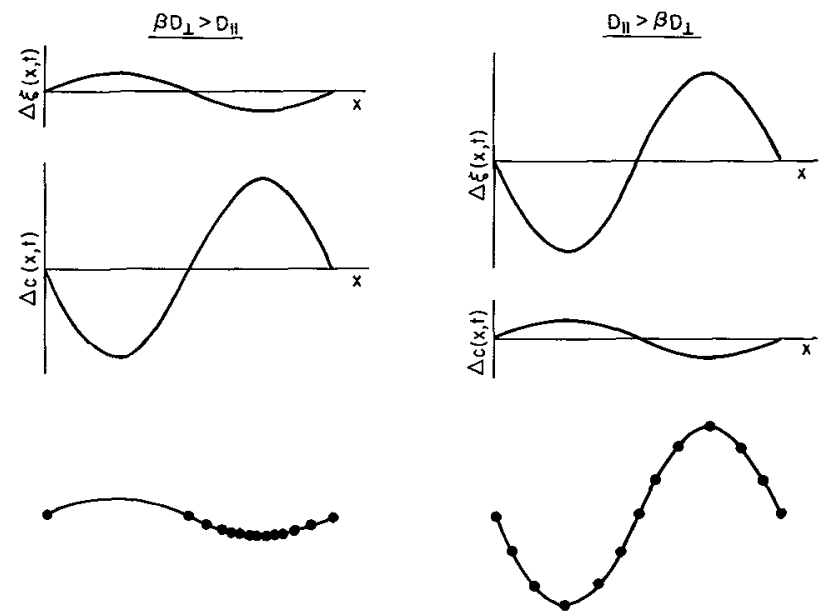

Frg. 5. - Schematic representation of variation of boundary displacement $\Delta \xi(x, t)$ and concentration profile $\Delta c(x, t)$ from that of a planar boundary as a function of spatial coordinate $x$. (a) Diffusion of impurities perpendicular to the boundary is much greater than diffusion parallel $\left(D_{\perp}>D_{\|}\right)$, and (b) diffusion of impurities parallel to the boundary is much greater than diffusion perpendicular $\left(D_{\|}>D_{\perp}\right)$.

maximum value of $v_{0}$ which satisfies this inequality $v_{1}$ is given by the expression

$$
v_{1}=k_{\mathrm{d}}\left(\beta D_{\perp} D_{\|}\right)^{1 / 2} .
$$

On the other hand, fluctuations characterized by wavelengths less than the mean impurity spacing $\lambda_{c}$ are not meaningful since at least one impurity must be associated with each periodic variation of boundary shape. Thus an upper limit for grain boundary velocity is given by the expression

$$
v_{2}=k_{\mathrm{c}}\left(\beta D_{\perp} D_{i \mid}\right)^{1 / 2},
$$

where $\quad k_{\mathrm{c}}=2 \pi / \lambda_{2}=2 \pi\left(N c_{0}\right)^{1 / 2}$. Therefore, a region of instability $\left(\lambda_{\mathrm{d}}>\lambda_{\mathrm{c}}\right)$ obtains under all conceivable experimental conditions.

It is interesting to note that $v_{2}$ corresponds closely to the condition for breakaway of a planar boundary from a uniform a uniform lateral excess concentration of impurities ; i. e., the driving force exceeeds the maximum value of the impurity drag force $F_{\mathrm{m}}$. In the present model $F_{\mathrm{m}}$ is given by the expression

$$
F_{\mathrm{m}}=2 \pi N c_{0}(\gamma \kappa T)^{1 / 2},
$$

which agrees within a factor of two with original expressions of Zener [6] (private communication) and Luicke and Detert [1]. Accordingly, it is likely that $v_{2}$ closely corresponds to the breakaway of a (non-planar) grain boundary from its excess impurity concentration.

A useful summary of the aforementioned discussion is presented in figure 6 , wherein macroscopic grain boundary velocity $v_{0}$ is plotted versus bulk impurity concentration for realistic values of material parameters. Three unique regions are evident : (1) a region labeled stable wherein a planar grain 


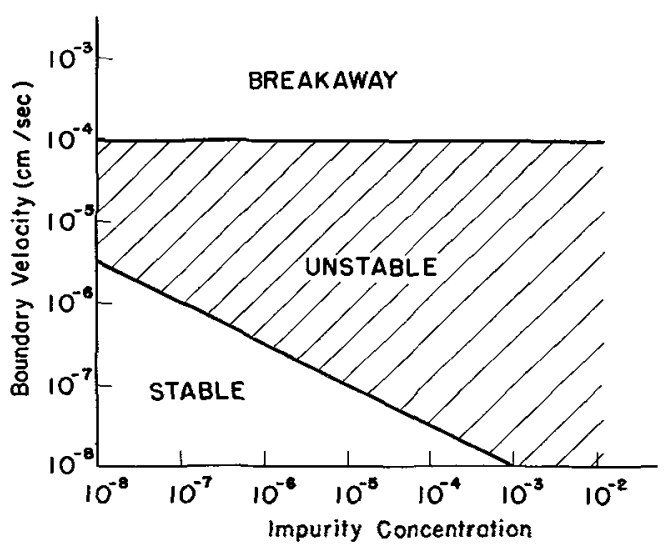

FIG. 6. - Macroscopic grain boundary velocity $v_{0}$ versus excess impurity concentration $c_{0}$. Regions of planar boundary stability, instability and breakaway are denoted for assumed values $2 \pi D_{1}=2 \pi D_{\|}=10^{-12} \mathrm{~cm}^{2} / \mathrm{s}, \quad \alpha=10, \quad N=10^{15} \mathrm{~cm}^{-2}$ and $\lambda_{\mathrm{d}}=10^{-2} \mathrm{~cm}$. Hatched area indicates region wherein a planar grain boundary shape and a uniform lateral impurity concentration profile are unstable.

boundary shape and a uniform lateral impurity profile are stable so that results of Lỉcke et al. and Cahn are valid, (2) a region labeled unstable wherein a planar grain boundary shape and a uniform lateral impurity profile are unstable so that results of Lücke et al. and Cahn are not valid, and (3) a region labeled breakaway wherein breakaway of a planar boundary from a uniform lateral concentration of impurities occurs. In general, a planar grain boundary characterized a uniform lateral concentration profile of impurities is the exception rather than the previously-assumed rule.

It must be emphasized that, due to the nature of the perturbation treatment utilized to solve Eqs. (2.3) and (2.4), it is only possible to demonstrate that a planar grain boundary shape and a uniform lateral impurity concentration profile are unstable when $v_{0}>v_{1}$. It can not be ascertained at present whether the boundary assumes stable nonplanar configurations or whether breakway occurs at much smaller values of $v_{0}$ than previously assumed ; i. e., values less than $v_{2}$. Therefore, figure 6 represents a composite chart including a line of demarcation separating regions of stable and unstable planar boundary shape and uniform concentration profile along the boundary as obtained from the analysis of Roy and Bauer (cf. Eq. 3.1) and a line denoting breakaway of a planar boundary from a uniform lateral concentration of impurities as obtained from the analyses of Lücke et al. and Cahn, which corresponds closely to an upper limit of macroscopic velocity in the present analysis (cf. Eq. 3.2). The perturbation analysis, however, provides no hint of breakaway even when amplitudes of boundary shape and concentration profile increase. Moreover, separation of regions of stability and instability is more diffuse than indicated in figure 6 due to the somewhat arbitrary criterion for instability in Eq. (2.7). Therefore, it is likely that grain boundaries assume stable nonplanar configurations prior to breakaway. Perhaps breakaway is characterized by some complex boundary shape or concentration profile wherein impurities are bypassed by a mechanism akin to dislocation bow out around impurities in a solid solution or to dendritic growth at a liquid-solid interface.

If stable nonplanar boundary shapes and nonuniform concentration profiles do obtain, a nonuniform distribution of impurities will be produced in the wake of the moving boundary, which, in the extreme case, may represent clusters or stringers of impurities. Thereafter these inhomogeneties will redistribute or redissolve in the matrix at a rate given by $k^{2} D_{\mathrm{m}}$, where $D_{\mathrm{m}}$ denotes the diffusion coefficient of impurities in the matrix. The kinetics of impurity redistribution could significantly affect reactions in the solid state and concomitant physical properties. For example, redistribution of impurities in the wake of a moving boundary could significantly affect the degree of solid solution strengthening, diffusion of impurities along the grain boundary could mark the onset of secondary recrystallization, or relative changes of $D_{\perp}$ and $D_{\|}$ with temperature could affect ultimate grain size and configuration. Further insight to these important phenomena, however, must await a more detailed analysis of stability of nonplanar grain boundary shapes and nonuniform impurity concentration profiles.

4. Summary. - Interaction of impurities with a moving grain boundary has been analyzed by a method which relaxes usual assumptions of a planar grain boundary shape and uniform lateral impurity concentration profile. In general, it is found that a moving boundary under the influence of impurities tends to assume nonplanar configurations characterized by a nonuniform lateral distribution of impurities. Boundary shape may fluctuate greatly with only slight redistribution of impurities or the impurity profile may fluctuate greatly with only slight variation of boundary shape. Likelihood of these processes depends on the relative degree of impurity diffusion parallel and perpendicular to the moving boundary. It is suspected that, in this unstable region, grain boundaries assume a spectrum of more complex morphologies and that elucidation of these morphologies can provide the first definitive description of the breakaway process and insight to more complex phenomena such as solid-solution strengthening, grain growth and secondary recrystallization.

Acknowledgement. - Financial support from the National Science Foundation is gratefully acknowledged. 


\section{References}

[1] LuCKe, K. and DeterT, K., Acta Met. 5 (1957) 628.

[2] CAHN, J. W., Acta Met. 10 (1962) 789.

[3] LUCKE, K. and STUWE, H. P., Recovery and Recrystallization of Metals L. Himmel ed., (Interscience, Wiley) 1963.
[4] Lucke, K. and Stuwe, H. P., Acta Met. 19 (1971) 1087. [5] Roy, A. and Bauer, C. L., Acta Met. 23 (1975) 957

[6] ZENER, C., private communication to SMITH, C. S., Trans. AIME 175 (1949) 15.

\section{DISCUSSION}

G. MARTIN : Do you think the main features of your solution would remain if you take into account the second dimension inside the grain boundary (3 dimensional problem rather than 2 dimensional one) ?
C. L. BAUER: The results will not change significantly. The wave number $k$ will merely be reselved into components $k_{x}$ and $k_{y}$ each exhibiting the same behaviour, at least within the limits of validity of the model. 